\section{A prevenção da incontinência urinária no contexto da pandemia da Covid-19: Ações educativas de uma Liga Acadêmica de Enfermagem}

\author{
The prevention of urinary incontinence in the \\ context of the Covid-19 pandemic: Educational \\ actions of an Academic Nursing League
}

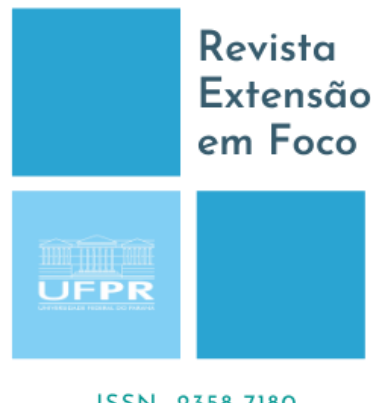

ISSN 2358.7180

\author{
Shirley Boller' ${ }^{1}$, Fernanda Moura D'Almeida Miranda ${ }^{2}$, Ana Paula Pereira ${ }^{3}$, Mariana \\ Kauling Barbosa ${ }^{4}$, Laura Alves Fachina ${ }^{5}$, Mahara Louíse Küchler ${ }^{6}$, Amanda Gabrielly \\ da Silva Kovalczykovski ${ }^{7}$, Andressa Blitzkow Scherer Teles ${ }^{8}$, Sarah Rebeca \\ Brueckheimer?
}

\begin{abstract}
RESUMO
Objetivo: descrever as ações realizadas pelos membros da Liga Acadêmica de Enfermagem em Estomaterapia de uma universidade pública do sul do Brasil na prevenção de Incontinência Urinária no contexto da pandemia da Covid-19. Método: trata-se de um relato de experiência sobre as divulgações científicas da temática de incontinência urinária que ocorreram no período de outubro de 2020 a março de 2021 por meio de plataformas digitais. Para tanto, foi realizado inicialmente a capacitação teórica, de forma remota, sobre incontinência urinária feminina e posteriormente elaborou-se e disseminou-se os informativos digitais. Resultados: Após a capacitação teórica, um folder informativo foi elaborado com ações preventivas e os principais cuidados com a incontinência urinária. Utilizou-se a rede social Instagram para veicular os informes sobre a temática. Ao total, foram compartilhadas seis informações baseadas em evidências científicas que foram elaborados pelos próprios membros da liga. A participação dos usuários foi identificada por meio de comentários. Em adição, foi estruturado um capítulo de livro voltado aos profissionais enfermeiros e acadêmicos de enfermagem na assistência aos pacientes incontinentes. Conclusão: O Instagram viabilizou, no período da pandemia, o compartilhamento de informações para a prevenção de incontinência urinária no público feminino da comunidade acadêmica
\end{abstract}

\footnotetext{
${ }^{1}$ Doutora em Enfermagem. Universidade Federal do Paraná (UFPR), Curitiba, Paraná, Brasil. E-mail: shirley.boller@ufpr.br. Orcid: https://orcid.org/0000-0001-8082-164X

${ }^{2}$ Doutora em Enfermagem. Universidade Federal do Paraná (UFPR), Curitiba, Paraná, Brasil. E-mail: fernandamiranda@ufpr.br. Orcid: https://orcid.org/0000-0001-7140-9557

${ }^{3}$ Graduanda em Enfermagem. Universidade Federal do Paraná (UFPR), Curitiba, Paraná, Brasil. E-mail: enfanapaulap@gmail.com. Orcid: https://orcid.org/0000-0003-1862-8982

${ }^{4}$ Graduanda em Enfermagem. Universidade Federal do Paraná (UFPR), Curitiba, Paraná, Brasil. E-mail: mariana.kaulingb@gmail.com. Orcid: https://orcid.org/0000-0002-8056-4708

${ }^{5}$ Graduanda em Enfermagem. Universidade Federal do Paraná (UFPR), Curitiba, Paraná, Brasil. E-mail: laurafachina@gmail.com. Orcid: https://orcid.org/0000-0002-5877-4872

${ }^{6}$ Graduanda em Enfermagem. Universidade Federal do Paraná (UFPR), Curitiba, Paraná, Brasil. E-mail: louisemahara@gmail.com. Orcid: https://orcid.org/0000-0002-7180-0625

${ }^{7}$ Graduanda em Enfermagem. Universidade Federal do Paraná (UFPR), Curitiba, Paraná, Brasil. E-mail: amanda.kovalczykovski@gmail.com. Orcid: https://orcid.org/0000-0001-7235-5407

${ }^{8}$ Graduanda em Enfermagem. Universidade Federal do Paraná (UFPR), Curitiba, Paraná, Brasil. E-mail: andressascherer3@gmail.com. Orcid: https://orcid.org/0000-0002-4407-375X

${ }^{9}$ Graduanda em Enfermagem. Universidade Federal do Paraná (UFPR), Curitiba, Paraná, Brasil. E-mail: sarah.rebeca@gmail.com. Orcid: https://orcid.org/0000-0001-9836-9364
} 
e externa a ela. O capítulo do livro motivou os membros da liga a se aproximar da temática e disseminar o conhecimento acadêmico de qualidade.

Palavras-chave: Integração Ensino-Pesquisa-Extensão. Atividades de Extensão. Educação para Prevenção.

\title{
ABSTRACT
}

\begin{abstract}
Objective: to describe the actions taken by the members of the Academic Nursing League in Stomatherapy of a public university in southern Brazil in the prevention of Urinary Incontinence in the context of the Covid-19 pandemic. Method: this is an experience report on the scientific disclosures on the subject of urinary incontinence that occurred from October 2020 to March 2021 through digital platforms. For this purpose, the theoretical training was initially carried out, remotely, on female urinary incontinence and later digital information was developed and disseminated. Results: After the theoretical training, an information folder was prepared with preventive actions and the main precautions with urinary incontinence. The social network Instagram was used to convey reports on the topic. In total, six pieces of information based on scientific evidence were shared and were elaborated by the league members themselves. User participation was identified through comments. In addition, a book chapter aimed at professional nurses and nursing students in assisting incontinent patients was structured. Conclusion: Instagram made it possible, in the period of the pandemic, to share information for the prevention of urinary incontinence in the female audience of the academic community and external to it. The chapter in the book motivated league members to approach the topic and disseminate quality academic knowledge.
\end{abstract}

Keywords: Teaching-Research-Extension Integration. Extension Activities. Prevention Education.

\section{INTRODUÇÃO}

O novo coronavírus (SARS-CoV-2), causador da doença Covid-19, foi responsável pela situação de pandemia, declarado pela Organização Mundial da Saúde (OMS) em 11 de março de 2020 (WHO, 2020). Com este cenário houve uma intensificação nas medidas de prevenção da Covid-19 que culminaram na interrupção do calendário acadêmico. Além disso, a crise sanitária impulsionou estratégias governamentais a fim de diminuir a transmissibilidade do vírus. Entre estas medidas está o isolamento social que impactou na realização das atividades desenvolvidas nas universidades. Assim, todos eventos presenciais da universidade foram suspensos e parte deles foram substituídos pela modalidade remota, por meio virtual, que se configurou como uma alternativa para a continuidade do desenvolvimento de tais ações (SAHU, 2020).

Neste contexto, a Liga Acadêmica de Enfermagem em Estomaterapia (LAENFE) da Universidade Federal do Paraná (UFPR), buscou desenvolver ações preventivas e educacionais voltadas à comunidade, promovendo o desenvolvimento de atividades 
científicas e permitindo aos acadêmicos o aprofundamento dos conteúdos e a aplicação na prática profissional. Os conteúdos abordados pela Laenfe em suas ações nas redes sociais e nas capacitações envolvem o cuidado com a pele, as incontinências urinária e fecal, ostomias e demais áreas abordadas na estomaterapia.

Um dos desafios para a Laenfe foi desenvolver ações à comunidade neste período pandêmico. Assim, foram planejadas atividades que pudessem ser desenvolvidas de maneira remota a fim de possibilitar ações educativas com material baseado em evidências científicas. Como tema para o planejamento das ações educativas optou-se pela Incontinência Urinária (IU).

A IU é definida como qualquer perda involuntária de urina (ABRAMS et al., 2017). É uma disfunção que afeta a qualidade de vida (QV), impactando diretamente em atividades diárias, no convívio em sociedade e na percepção da própria saúde, principalmente relacionados ao bem-estar social e mental (CESTÁRI; SOUZA; SILVA, 2017).

Esta disfunção é um grave problema de saúde pública que acomete ambos os sexos de diferentes idades e de todos os níveis socioeconômicos. Calcula-se que aproximadamente 400 milhões de pessoas no mundo são afetadas. No Brasil, dados estimam que há 10 milhões pessoas com incontinência (OLIVEIRA et al., 2018).

A IU é frequente entre as mulheres que enfrentam risco aumentado face ao processo de envelhecimento, obesidade, parto vaginal, presença de Doença Pulmonar Obstrutiva Crônica, Diabetes mellitus, entre outras comorbidades (PEDERSEN et al., 2017).

Com base no impacto causado pela Incontinência Urinária na QV das pessoas acometidas, e, somando-se isso às dificuldades enfrentadas decorrentes da pandemia, que complicaram o acesso aos serviços de saúde, pelos usuários, e a paralização das atividades universitárias, tornou-se relevante disseminar as informações cientificas no meio virtual. Sendo assim o presente relato de experiência tem por objetivo descrever as ações realizadas pelos membros da Laenfe de uma universidade pública do sul do Brasil na prevenção de Incontinência Urinária no contexto pandêmico.

\section{MÉTODO}


Trata-se de um estudo descritivo, com abordagem qualitativa, do tipo relato de experiência. O período foi de outubro de 2020 a março de 2021. Participaram deste relato os membros da Laenfe: ligantes, gestores, professores e profissionais enfermeiros. Os locais para o desenvolvimento das ações educativas foram a plataforma Google Meet e a rede social Instagram para os compartilhamentos de informações científicas a comunidade.

Para o desenvolvimento das atividades da Laenfe optou-se por quatro fases, que foram baseadas nos princípios norteadores das atividades de extensão sendo eles: “Impacto e Transformação; Interação Dialógica; Interdisciplinaridade; Indissociabilidade e Impacto na Formação de estudante, e visa resultado de mútuo interesse, para a sociedade e para a comunidade acadêmica" (SEPT, 2021).

Na primeira fase, com o intuito de subsidiar a elaboração dos produtos decorrentes das ações ligadas à prevenção da IU, os membros da Laenfe participaram de uma atualização na temática com uma Enfermeira Estomaterapeuta, expertise em cuidados com IU no público feminino. Devido à pandemia da Covid-19 as aulas da atualização foram realizadas no modelo expositiva-dialogada, de forma remota, por meio da plataforma Google Meet, realizada em três dias contabilizando uma carga horária total de 04 horas.

Durante o período das aulas, os participantes foram estimulados a desenvolver o pensamento crítico-reflexivo no que concerne as competências do enfermeiro durante a realização da consulta de enfermagem para pacientes com incontinência. Um material com o conteúdo apresentado durante a aula elaborado pela Enfermeira Estomaterapeuta foi disponibilizado aos estudantes previamente, e com autorização da Enfermeira as aulas foram gravadas e compartilhadas com os membros da liga.

$\mathrm{Na}$ segunda fase confeccionou-se um folder informativo sobre a temática, com o objetivo de atingir o público alvo feminino interno e externo à universidade. O material foi elaborado pelos membros da Laenfe com base em evidências científicas. Foi realizada uma revisão bibliográfica, seguida da escolha do design gráfico, o layout, diagramação e adequação da linguagem ao seu público-alvo, durante os meses de outubro a dezembro de 2020.

$\mathrm{Na}$ terceira fase optou-se por promover a divulgação de conteúdos relacionados à temática por meio da rede social Instagram, com a finalidade de alcançar o maior número de pessoas do sexo feminino. As postagens foram elaboradas pelos ligantes e o conteúdo abordado em cada publicação foi decidido em reunião. Após finalização do material, as 
postagens foram reavaliadas pelos demais integrantes da Laenfe. Uma vez aprovado, os conteúdos foram sendo publicados na rede social, de acordo com um cronograma previamente elaborado.

$\mathrm{Na}$ quarta fase foi produzido um capítulo de livro, designado a subsidiar o processo de enfermagem durante a consulta de enfermeiros e acadêmicos, com informações sobre o diagnóstico de enfermagem e o manejo dos principais tipos de IU feminina. Para produção do material participaram todos os membros da Laenfe, incluindo ligantes, gestores, professores e os colaboradores com expertise na temática. O conteúdo foi selecionado por meio de uma revisão bibliográfica e elencou-se as principais evidências científicas para incorporar as orientações ao público-alvo do material. Este material encontra-se aceito para publicação.

Os colaboradores e professores que participaram da redação e revisão do todos os materiais produzidos pela Laenfe, contribuíram com sua experiência e aperfeiçoamento do conteúdo, visto que são profissionais especializados em estomaterapia.

\section{RESULTADOS E DISCUSSÃO}

Foram realizadas quatro ações educativas sendo elas: atualização teórica sobre IU feminina, folder informativo, divulgação de orientações na rede social Instagram e capítulo de um livro. Essas ações são apresentadas detalhamento a seguir.

A atualização referente a Consulta de Enfermagem a mulher com IU ocorreu em três módulos. Estavam presentes os doze membros da Laenfe. O conteúdo abordado estava relacionado a anatomia e fisiologia do sistema urinário, a diferença entre IU, patologias que afetam frequência urinária, os três tipos principais de IU e suas características, tratamentos, prevenções, exame físico e as etapas da consulta de enfermagem. Os participantes ao final dos três módulos foram avaliados por meio de uma discussão de caso clínico com a finalidade de definir o diagnóstico e a melhor indicação de tratamento para a disfunção.

Qualquer assistência realizada pela enfermagem deve estar baseada no método científico do Processo de Enfermagem, sendo assim Dantas, Santos e Tourinho (2016) em seus estudos, afirmam que a Consulta de Enfermagem na Atenção Primária à Saúde é uma combinação entre o conhecimento humano, científico e empírico, que organiza a 
atividade do enfermeiro, tendo como objetivo fornecer uma assistência de qualidade e que se confirma no cuidado a comunidade. Portanto, durante a Consulta de Enfermagem, o profissional pode detectar os impactos na qualidade de vida do incontinente e assim buscar o melhor tratamento para o paciente (DAGOSTIN et al., 2020).

Para alcançar o propósito proposto pelas Diretrizes Curriculares Nacionais para os cursos da área de saúde publicados a partir de 2001 (BRASIL, 2001), que recomendam a integração entre ensino, pesquisa, extensão e a assistência com atitudes voltadas para a comunidade, se deve ter um currículo que estimule o ensino-aprendizagem do estudante para que em seu futuro profissional ele saiba vincular o seu conhecimento adquirido durante sua formação e as necessidades dos pacientes (ARAUJO et al., 2019). Nesse sentido, a atualização aos estudantes possibilita aprimoramento sobre a temática e contribui para uma melhor orientação aos pacientes e comunidade sobre os cuidados e prevenções da IU, garantindo qualidade ao cuidado com a população.

Estudo realizado por Gusso e colaboradores (2020) afirma que os estudantes adquirem competências e habilidades por meio da formação com o intuito de orientar a comunidade a partir do desenvolvimento de condutas que aumentem a aptidão e o êxito em suas intervenções. Corroborando com esse estudo, destaca-se que a capacitação é importante para elaboração de novos métodos educativos sobre IU.

Outra ação educativa desenvolvida pela Laenfe foi o folder informativo. Este foi desenvolvido no tamanho A4 e dividido em 6 sessões. Seu design gráfico foi formado com base nas cores utilizadas pela Laenfe (azul, laranja e branco), aplicando recursos visuais, como círculos e desenhos, para atrair os leitores e linguagem apropriada para o público, na intenção de que o informativo seja mais atraente. O tema foi abordado de forma clara e objetiva, pois se trata de um instrumento breve de comunicação e fácil entendimento. A fonte utilizada e seu tamanho foram escolhidos de forma adequada, em que fosse possível a leitura sem esforços e que não prejudicasse o conteúdo.

O material, intitulado "Precisamos falar sobre incontinência urinária", foi produzido através do programa Canva. Os tópicos abordados no instrumento foram: o que é incontinência urinária, fisiologia da micção, fatores de risco, principais sinais e sintomas, prevenção, locais para procurar ajuda e dados de prevalência.

$\mathrm{Na}$ capa do folder foi apresentado o informativo, com seu título e os logotipos da universidade e da Laenfe. Ao abrir encontra-se primeiramente as informações em forma de texto sobre o que é a IU e qual a fisiologia da micção. Em seguida em tópicos são apresentados os principais fatores de riscos e sintomas. 
A próxima sessão exibe as formas de prevenção de IU e os locais que podem ser buscados para solicitar atendimento, divulgando a casa 3 da UFPR e as Unidades Básicas de Saúde do município de Curitiba. Por último encontra-se os dados de prevalência, os autores do material e as referências utilizadas. Como apresentado nas figuras 1 e 2 .

Figura 1 - Primeiro segmento do folder

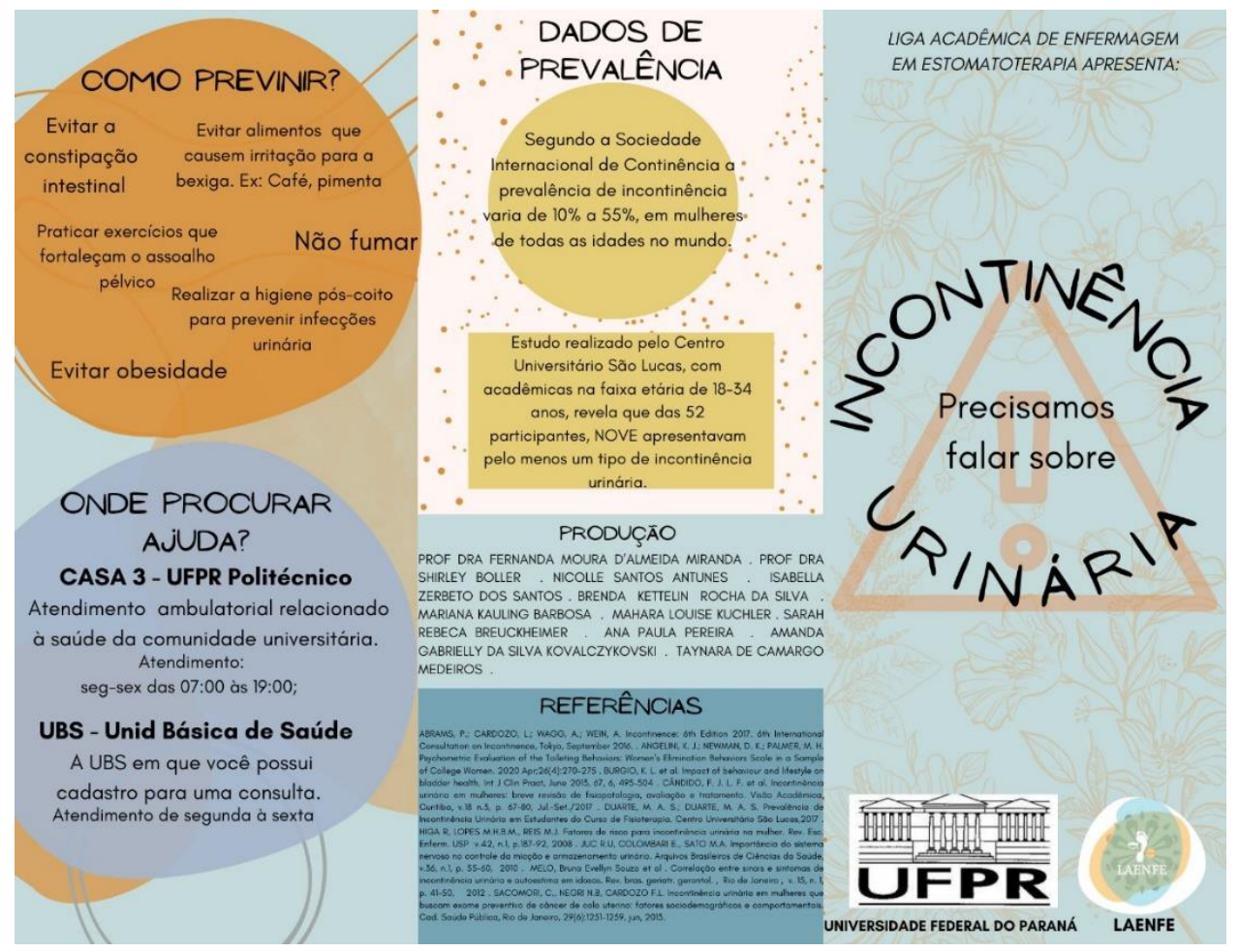

Fonte: Autoria própria (2020).

Figura 2 - Segundo segmento do folder 


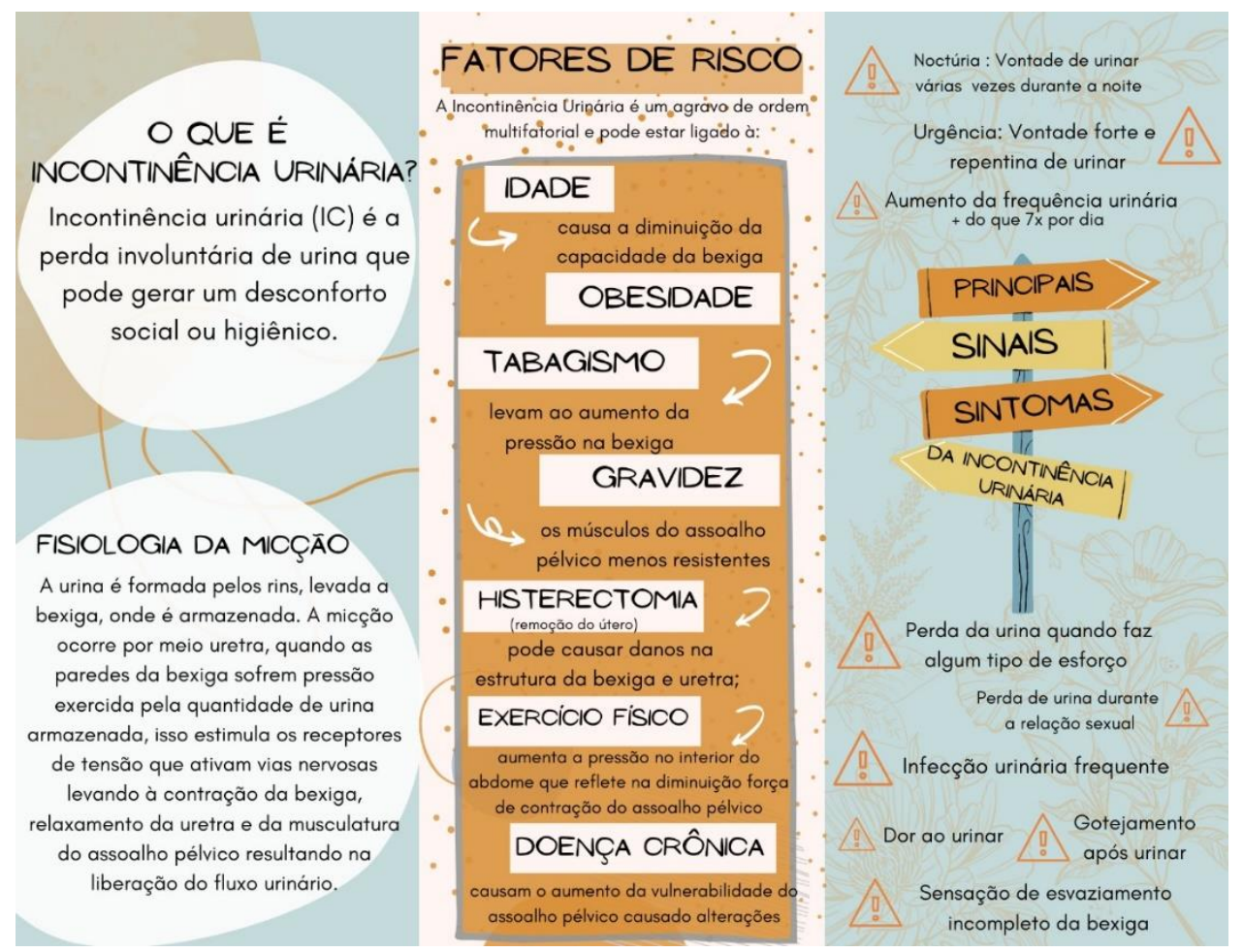

Fonte: Autoria própria (2020)

Após a aprovação do folder pelas coorientadoras e pela enfermeira especialista, o material será divulgado através das redes sociais e será realizada a impressão do instrumento para que no retorno das atividades presenciais o mesmo possa ser entregue ao público-alvo.

Com o intuito de apoiar as orientações verbais, além de apresentar imagens e ilustrações, os folders têm se destacado. As ações de Educação em Saúde (ES) precisam ser realizadas através de orientações claras, adequadas e objetivas (ANDRADE et al., 2019). A ES busca estimular a participação do indivíduo no seu próprio cuidado diário e não apenas em casos de adoecimento (FEITOSA et al., 2019), visível para o aprimoramento das práticas nocivas à saúde da população (MOREL, 2020). Com isso, o desenvolvimento do folder teve a intenção de alertar o público-alvo sobre a importância da temática e apresentar brevemente sobre a mesma, pois este ainda é um assunto pouco abordado.

Na terceira fase foram realizadas as publicações no Instagram com o objetivo de facilitar o acesso as informações durante o período de isolamento. A divulgação na rede social alcança a população alvo e propicia informações baseadas em evidências científicas sobre os cuidados referentes a IU, com posts interativos - usando de ferramentas que a plataforma oferece para a interação com a comunidade por meio de 
caixas de perguntas, enquetes, testes rápidos nos stories e por comentários nos posts. Essas ferramentas proporcionam uma interação com a comunidade, permitindo que essa possa esclarecer dúvidas e receber informação com qualidade.

A primeira publicação sobre incontinência urinária foi uma breve contextualização sobre o assunto, com sua definição, e ressaltando o quanto ela afeta a qualidade de vida do paciente, e epidemiologia, explicando quais os grupos mais acometidos. Ver figura 3 e 4.

Figura 3 - Definição de Incontinência Urinária

Você sabe o que é Incontinênci215

Urinária (IU)?

- De acordo com a International

Continence Society (ICS)

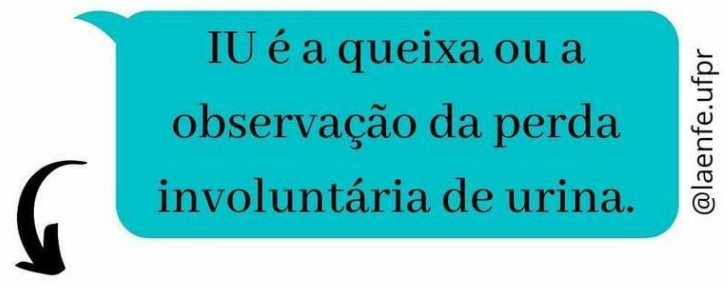

Esta perda pode gerar

desconforto social ou higiênico.

Fonte: Autoria própria (2020).

Figura 4 - Epidemiologia da Incontinência Urinária

E você sabe quais são os grupos d $3 / 5$ pessoas acometidas pela IU?

- De 1.894 mulheres $48,3 \%$

apresentaram IU, sendo 34

delas jovens entre 18-29 anos;

- De 477 homens com cerca de 61 anos, 51\% apresentaram;

- De 401 idosos ( $\geq 65$ anos) $32,2 \%$ tinham IU;

- De 2.750 crianças $8,6 \%$ entre 11 e 14 anos, a maioria meninos.

Fonte: Autoria própria (2020). 
As divulgações subsequentes foram relacionadas aos possíveis agravamentos dos sintomas do trato urinário inferior, tendo como exemplo a forma incorreta da utilização dos banheiros públicos por preocupação com a limpeza dos mesmos, exemplo na figura 5 .

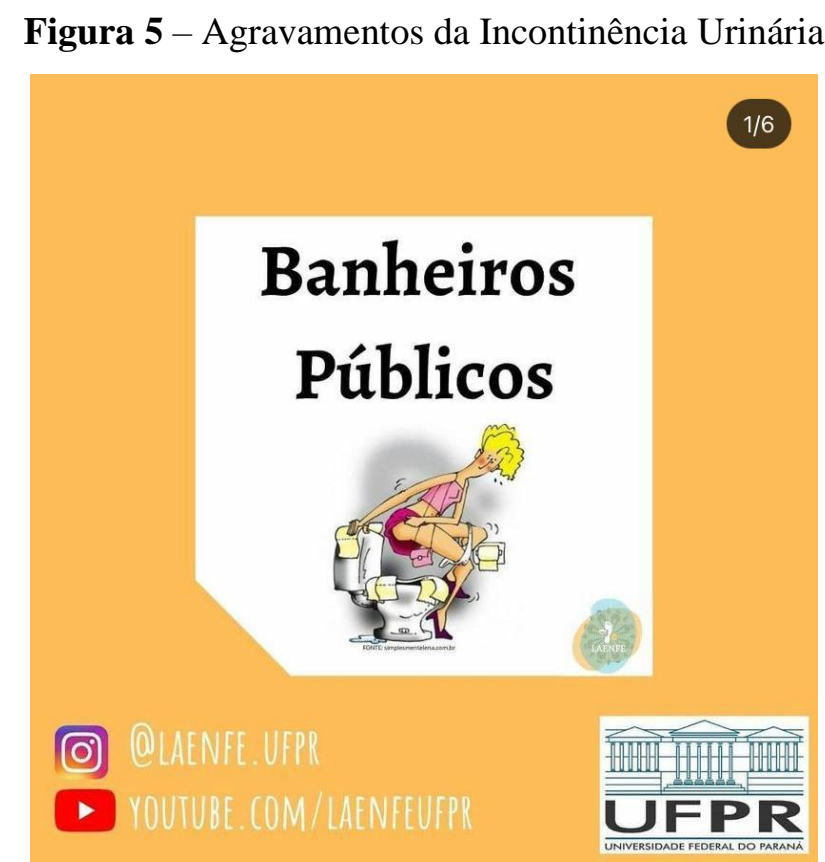

Fonte: Autoria própria (2020).

Com fins de esclarecimentos de suas diferenças para que assim possam ajudar na orientação do tratamento dessa incontinência, as classificações de IU foram publicadas em seguida (figura 6).

Figura 6 - Classificações da Incontinência Urinária 


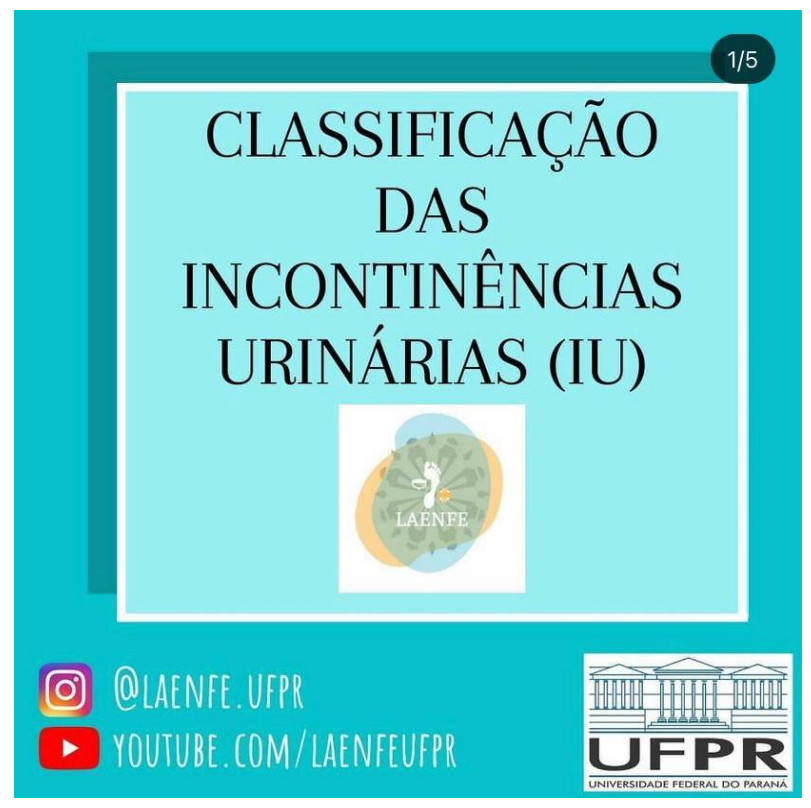

Fonte: Autoria própria (2020).

Para o melhor entendimento sobre o assunto e de uma forma sucinta, os ligantes, utilizando ferramentas disponíveis na plataforma de comunicação, também fizeram um pequeno vídeo explicativo referente à fisiologia da micção, para que assim ficasse mais esclarecido sobre quais órgãos podem estar envolvidos no processo da incontinência juntamente ao sistema nervoso envolvido no processo miccional (figura 7).

Figura 7 - Fisiologia da Micção

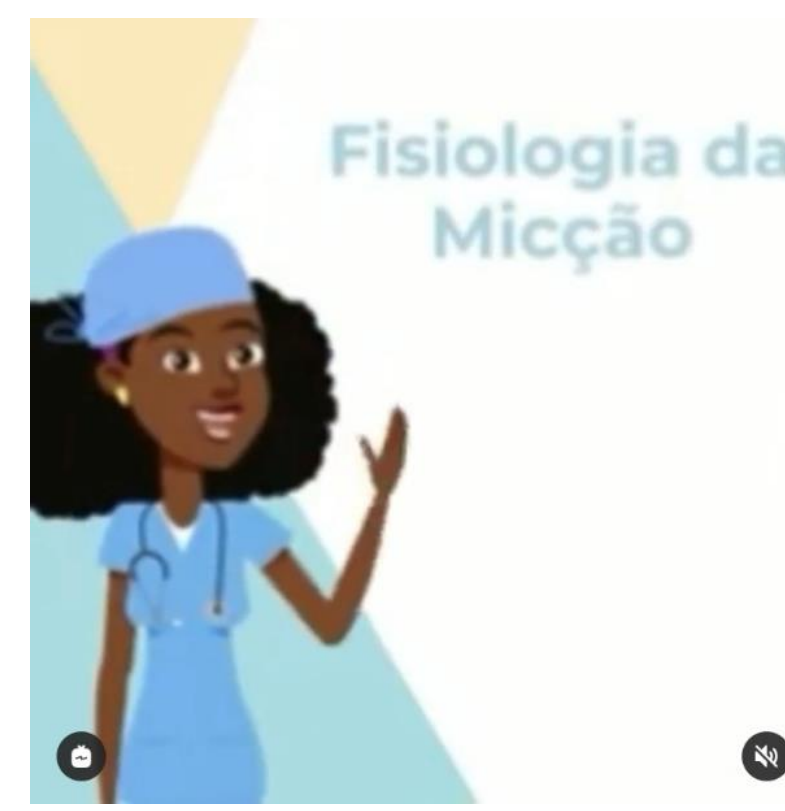

Fonte: Autoria própria (2020). 
Para que o público-alvo pudesse ficar atento aos sintomas, eles foram apresentados em seguida, apontando não somente sintomas fisiológicos, mas também sobre os fenômenos relacionados a vida social (figura 8).

Figura 8 - Sintomas da Incontinência

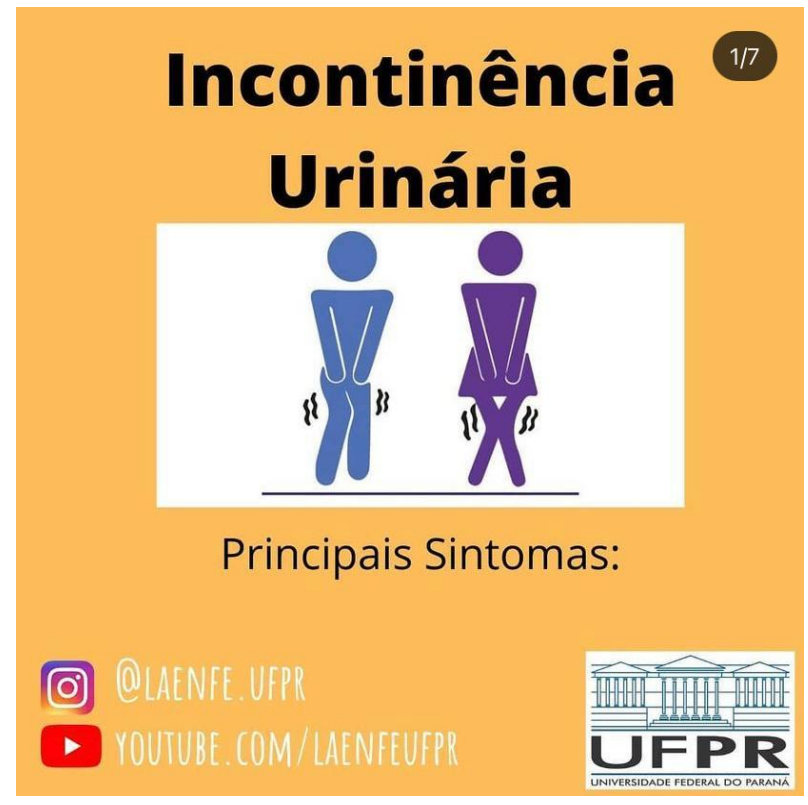

Fonte: Autoria própria (2021).

Sabendo da importância de pensar em prevenir os problemas da musculatura do assoalho pélvico, mudando os hábitos de somente se atentar as disfunções quando os sintomas aparecem, os estudantes fizeram uma publicação sobre os cuidados necessários para a prevenção da IU, como visto na figura 9.

Figura 9 - Prevenção da Incontinência Urinária

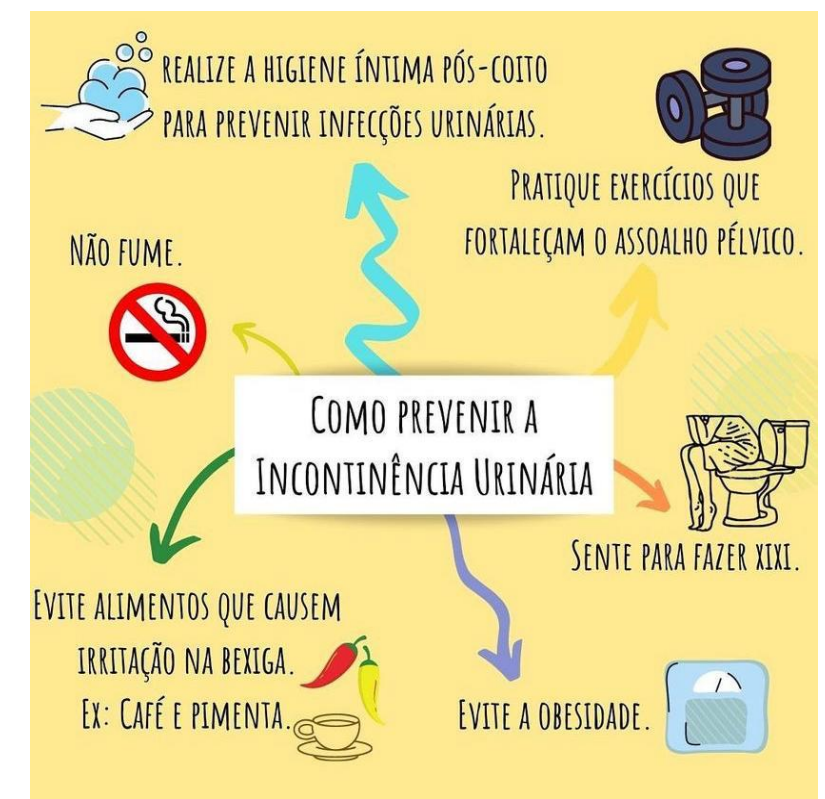

Fonte: Autoria própria (2021). 
E para esclarecer ao público que a IU tem tratamento, na publicação foi relatado todas os recursos terapêuticos disponíveis, como os tratamentos não invasivos, os medicamentosos e o tratamento cirúrgico (figura 10).

Figura 10 - Tratamento Incontinência Urinária

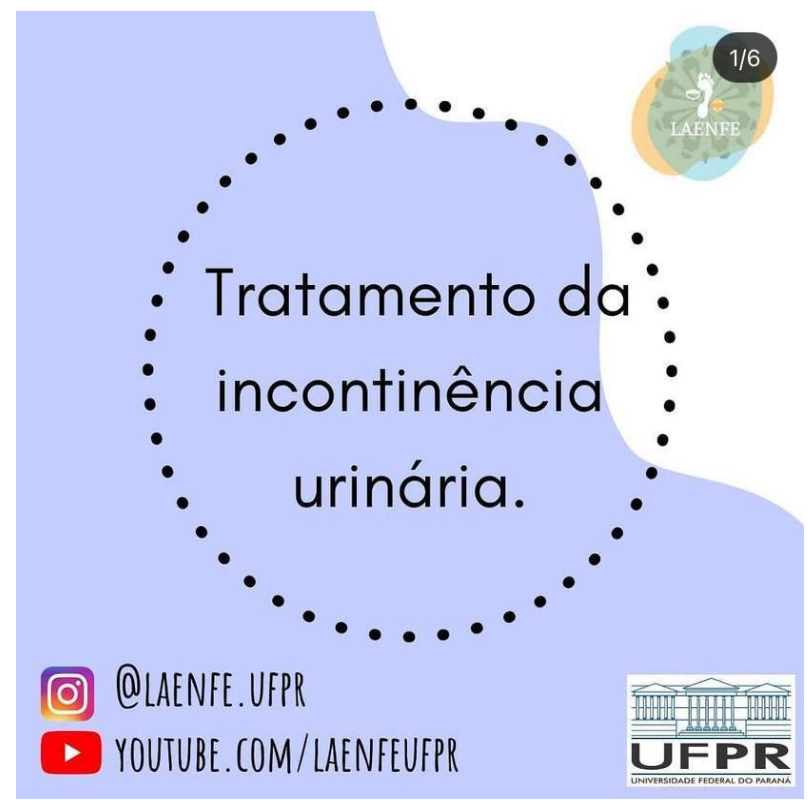

Fonte: Autoria própria (2021).

Os ligantes responsáveis pelas publicações na rede social sentiram a necessidade de compartilhar esse conhecimento com à comunidade. Conforme aponta Oliveira e colaboradores (2018) essa disfunção afeta drasticamente a vida do incontinente, o que torna importante o debate e as orientações com a comunidade sobre essa temática.

Assim, a educação em saúde permite a pessoa afetada compreender os processos de assimilação, enfrentamento, recuperação, adaptação e aceitação ao tratamento. Neste contexto, o enfermeiro desempenha um papel fundamental para com o paciente a fim de orientá-lo sobre a prevenção, a disfunção, o tratamento e suas consequências para a qualidade de vida deste (VALENÇA et al., 2016). Destaca-se a importância da participação dos ligantes neste contexto, devido eles serem futuros profissionais que podem contribuir para a melhoria da assistência em saúde em seus diversos cenários.

A última fase das ações educativas realizadas foi a elaboração de um capítulo de livro intitulado "O processo de enfermagem no cuidado ao paciente com incontinência urinária", voltado aos enfermeiros e aos acadêmicos de enfermagem. Este capítulo apresenta questões relevantes a temática, com objetivo de auxiliar os profissionais e os acadêmicos na assistência de enfermagem com os pacientes incontinentes. 
A introdução do capítulo aborda informações sobre o conceito das Ligas Acadêmicas, a Sistematização da Assistência de Enfermagem (SAE), e a definição de IU e suas classificações. No desenvolvimento do capítulo é exposto a epidemiologia da IU, seguido pela classificação das incontinências, abordando os principais tipos, como Incontinência Urinária por esforço (IUE), Incontinência Urinário por Urgência (IUU) e Incontinência Urinária Mista (IUM). No decorrer do material são abordados os principais sinais e sintomas e os impactos ocasionados pela IU.

Quanto aos tipos de tratamentos foram abordados o tratamento cirúrgico, o tratamento de reabilitação da Musculatura do Assoalho Pélvico (MAP), tratamento medicamentoso e o tratamento comportamental. No capítulo foi desenvolvido um roteiro para o processo de enfermagem no cuidado ao paciente incontinente, dividido em 4 etapas.

A primeira etapa é definida pela coleta de dados de enfermagem. A segunda são os diagnósticos de enfermagem. A terceira etapa aborda o planejamento/intervenção de enfermagem. E a quarta etapa abrange a avaliação de enfermagem. Por fim o material apresenta as considerações finais e as referências utilizadas. Este capítulo foi aceito para publicação neste semestre.

A elaboração do capítulo de um livro proporciona ao estudante o aprofundamento conceitual relacionado a temática selecionada. Ainda, o estudante é desafiado a refletir e discutir criticamente sobre o tema. Nesse contexto, o processo formativo deve abranger o progresso de habilidades e competências, proporcionando uma aprendizagem crítica, reflexiva, autônoma e continuada (COLARES; OLIVEIRA, 2020).

As Diretrizes Curriculares Nacionais orientam as condições para a formação e capacitação de um profissional, para que esteja apto a atuar nos serviços de saúde. Observa-se que as transformações sociais do processo saúde-doença sofrem constantes modificações, isso acaba interferindo na formação acadêmica. Para essas transformações e para a demanda dos serviços de saúde, é preciso ter um conjunto de habilidades e competências gerais, uma delas é a Educação Permanente dos estudantes e profissionais (SAMPAIO et al., 2019). Com isso, a confecção do material didático é muito importante para a atualização de enfermeiros e acadêmicos de enfermagem, servindo como base de conhecimento para a assistência de enfermagem aos pacientes incontinentes.

\section{CONCLUSÃO}


No ano de 2020/21 as atividades da Laenfe foram executadas de forma remota, face a restrição imposta pela pandemia da COVID-19. A participação ativa dos ligantes, gestores, professores e profissionais enfermeiros, viabilizaram a promoção de ações extensionistas relacionadas disseminação de informações científicas da prevenção da IC feminina, por meio virtual.

Por se tratar de uma ferramenta de fácil acesso, o instagram abrange um público amplo e muitas vezes interessados em informações de qualidade. E nesse sentido, as ações educativas da Laenfe, além de proporcionar aos estudantes um aprofundamento teórico e respaldado em evidência cientifica sobre o cuidado de enfermagem ao paciente com IU, possibilitaram também a visibilidade da temática entre os usuários da rede social da liga.

A construção do capítulo do livro concedeu aos membros da Laenfe uma compreensão aprofundada da temática e proporcionou a apreciação da escrita técnica e científica, tão essencial para disseminar o conhecimento acadêmico de qualidade.

Ainda que pandemia tenha suspendido as ações presenciais planejadas pela Laenfe, a utilização das tecnologias da informação permitiu a continuidade das atividades demonstrando o compromisso extensionista com a formação de futuros profissionais enfermeiros e contribuindo na disseminação de informações cientificas e seu papel social frente à comunidade.

\section{REFERENCIAS}

ABRAMS, P. et al. (Eds) Incontinence 6th Edition (2017). Bristol: ICI-ICS. International Continence Society, 2017.

ANDRADE, L. M. et al. Construção de folder educativo para pacientes ambulatoriais com insuficiência cardíaca: relato de experiência. Congresso Internacional de Enfermagem, v. 1, n. 1, Maio 2019. Disponível em: <https://eventos.set.edu.br/cie/article/view/11261/4375>. Acesso em: 13 abr. 2021.

ARAUJO, C. R. de C. e. et al. Contribuição das Ligas Acadêmicas para formação em Enfermagem. Enferm. Foco, v. 10, n. 6, p. 137-142, 2019. DOI 10.21675/2357707X.2019.v10.n6.2802. Disponível

em: 
<http://revista.cofen.gov.br/index.php/enfermagem/article/view/2802>. Acesso em: 15 abr. 2021.

BRASIL. Resolução $\mathrm{n}^{\circ}$ 3, de 7 de novembro de 2001. Institui Diretrizes Curriculares Nacionais do Curso de Graduação em Enfermagem. Diário Oficial da União, Brasília, Seção 1, p. 37, 09 Nov 2001. Disponível em: < http://portal.mec.gov.br/cne/arquivos/pdf/CES03.pdf>. Acesso em: 07 abr. 2021.

CESTÁRI, C. E., SOUZA T. H. C., SILVA A. S. Impacto da incontinência urinária na qualidade de vida de idosas. Revista Ciência e Estudos Acadêmicos de Medicina, Universidade do Estado de Mato Grosso - UNEMAT (Cáceres), n. 7, p. 27 37 , Jan.-Jul. 2017.

Disponível

em: <https://periodicos.unemat.br/index.php/revistamedicina/article/viewFile/1773/2091>. Acesso em: 06 abr. 2021.

COLARES, K. T. P.; OLIVEIRA, W. de. Uso de metodologias ativas sob a ótica de estudantes de graduação em Enfermagem. Revista SUSTINERE, Rio de Janeiro, v. 8, n. 2, p.374-394, Jul-Dez. 2020. DOI 10.12957/sustinere.2020.45088. Disponível em: <https://www.e-publicacoes.uerj.br/index.php/sustinere/article/view/45088/36343>. Acesso em: 13 abr. 2021.

DAGOSTIN, V. S. et al. Processo de enfermagem aplicado na atenção à saúde da pessoa com incontinência urinária e fecal. Braz. J. Hea. Rer., Curitiba, v. 3, n. 5, p. 11496-11508, Set. 2020. DOI 10.34119/bjhrv3n5-011. Disponível em: <https://www.brazilianjournals.com/index.php/BJHR/article/view/16040/13132>. Acesso em: 15 abr. 2021.

DANTAS, C. N.; SANTOS, V. E. P.; TOURINHO, F. S. V. A consulta de enfermagem como tecnologia do cuidado à luz dos personagens de Bacon e Galimberti. Texto contexto - enferm., Florianópolis, v. 25, n. 1, e2800014, Mar. 2016. DOI 10.1590/0104-0707201500002800014. Disponível em: $<$ http://www.scielo.br/scielo.php?script=sci_arttext\&pid=S010407072016000100601\&lng=pt\&nrm=iso>. Acesso em 15 abr. 2021.

FEITOSA, A. L. F. et al. Sala de espera: estratégias de educação em saúde no contexto da atenção básica. Rev. Bra. Edu. Saúde, v. 9, n. 2, p. 67-70, Abr-Jun, 2019. DOI 10.18378/rebes.v9i2.6401 Disponível em: <https://www.editoraverde.org/gvaa.com.br/revista/index.php/REBES/article/view/6401 /5612>. Acesso em: 14 abr. 2021. 
GUSSO, H. L. et al. Ensino superior em tempos de pandemia: diretrizes à gestão universitária. Educ. Soc., Campinas, v. 41, e238957, Set. 2020. DOI 10.1590/es.238957. Disponível em: <http://www.scielo.br/scielo.php?script=sci_arttext\&pid=S010173302020000100802\&lng=en\&nrm=iso>. Acesso em: 07 abr. 2021.

MOREL, A. P. M. Da educação sanitária à educação popular em saúde: reflexões sobre a pandemia do coronavirus. Revista Estudos Literários, v.2, n.3. 2020. Disponível em: 〈https://revistas.ufrj.br/index.php/estudoslibertarios/article/viewFile/34114/19178>. Acesso em: 14 abr. 2021.

OLIVEIRA, L. G. P. et al. A. Incontinência urinária: a atuação do profissional de enfermagem. Revista Eletrônica Acervo Saúde, n. 18, p. e118. Dez. 2018. DOI 10.25248/reas.e118.2019. Disponível em: <https://acervomais.com.br/index.php/saude/article/view/118>. Acesso em: 07 abr. 2021.

PEDERSEN, L. S. et al. Prevalence of urinary incontinence among women and analysis of potential risk factors in Germany and Denmark. Acta Obstet Gynecol Scand, v. 96, n. 8, p. 939-948, 2017. DOI 10.1111/aogs.13149. Disponível em: <https://obgyn.onlinelibrary.wiley.com/doi/epdf/10.1111/aogs.13149>. Acesso em: 06 abr. 2021.

SAHU, P. Closure of Universities Due to Coronavirus Disease 2019 (COVID-19): Impact on Education and Mental Health of Students and Academic Staff. Cureus, v. 12, n. 4, p. 1-6, 2020. DOI 10.7759/cureus.7541. Disponível em: <https://www.ncbi.nlm.nih.gov/pmc/articles/PMC7198094/>. Acesso em: 14 abr. 2021.

SAMPAIO, G. B. et al. Educação Permanente e o Processo Formativo dos Profissionais da Saúde: Entrelace de Saberes. Revista Eletrônica Acervo Saúde, n. 25, p. 1-8, Jun. 2019. DOI 10.25248/reas.e630.2019. Disponível em: <https://acervomais.com.br/index.php/saude/article/view/630/500>. Acesso em: 13 abr. 2021.

SEPT - Setor de Educação Profissional e Tecnológica. O que é Extensão. SEPT/UFPR，2021. Disponível em: <http://www.sept.ufpr.br/portal/entendendo-aextensao>. Acesso em: 07 abr. 2021. 
VALENÇA, M. P. et al. Cuidados de Enfermagem na Incontinência Urinária: um Estudo de Revisão Integrativa. ESTIMA, v. 14 n. 1, p. 43-49, 2016. DOI 10.5327/Z18063144201600010007. Disponível em: <https://www.revistaestima.com.br/estima/article/view/195>. Acesso em: 07 abr. 2021. WHO - WORLD HEALTH ORGANIZATION. WHO Director-General's opening remarks at the media briefing on COVID-19 - 11 March 2020. Mar. 2020. Disponível em: <https://www.who.int/director-general/speeches/detail/who-directorgeneral-s-opening-remarks-at-the-media-briefing-on-covid-19---11-march-2020> Acesso em: 16 abr. 2021.

Recebido em: 16 de abril de 2021. Aceito em: 20 de abril de 2021. 